\title{
Endoscopic ablation of Hunner's lesions in interstitial cystitis patients
}

\author{
Ryan A. Payne, MD; R. Corey O'Connor, MD; Margarita Kressin, MD; Michael L. Guralnick, MD, FRCSC
}

See related article on page 478

\begin{abstract}
Introduction: We report our experience with endoscopic ablation of Hunner's lesions in women with interstitial cystitis (IC).

Methods: A chart review was performed on 14 patients with IC symptoms who were identified to have bladder lesions and underwent endoscopic ablation. A Hunner's lesion was identified as an area of erythema that reproduced the patients' pain when touched by the cystoscope. Pathology reports were reviewed and improvement in pain was used as the main outcome measure.

Results: Of the 14 patients, 12 had more than 50\% symptomatic improvement and 8 patients reported $100 \%$ improvement. Mean improvement was $76 \%$. In all patients who improved, the biopsy specimen showed inflammatory cystitis, often with epithelial denudation. Four patients had symptomatic recurrence, but all had improvement after repeat ablation.

Conclusion: Endoscopic ablation of Hunner's lesions improves symptoms in IC patients. Recurrence of symptoms should prompt repeat cystoscopy to identify recurrent lesions, as repeat ablation offers symptomatic improvement.
\end{abstract}

Can Urol Assoc J 2009;3(6):473-7

\section{Résumé}

Introduction : L'article fait état de notre expérience concernant I'ablation endoscopique d'ulcères de Hunner chez des femmes atteintes de cystite interstitielle $(\mathrm{Cl})$.

Méthodologie : On a mené un examen des dossiers de 14 patientes présentant des symptômes de $\mathrm{Cl}$ chez qui des lésions vésicales avaient été observées et traitées par ablation endoscopique. Un ulcère de Hunner était défini comme une zone d'érythème reproduisant la douleur décrite par la patiente lorsque cette zone était touchée à l'aide du cystoscope. Les rapports de pathologie ont été examinés et la réduction de la douleur a été utilisée comme principal critère d'évaluation.

Résultats : Sur les 14 patientes, 12 ont présenté une réduction supérieure à $50 \%$ des symptômes et 8 ont signalé une réduction de $100 \%$. Le taux moyen de réduction était de $76 \%$. Chez toutes les patientes ayant signalé une réduction des symptômes, l'échantillon de tissu prélevé par biopsie montrait la présence de cystite inflammatoire, souvent accompagnée de dénudation épithéliale. Quatre patientes ont signalé une réapparition des symptômes, mais une nouvelle ablation a permis de réduire encore une fois les symptômes.
Conclusion : L'ablation endoscopique des ulcères de Hunner entraîne une réduction des symptômes en présence de $\mathrm{Cl}$. La réapparition des symptômes devrait être prise en charge par une nouvelle cystoscopie afin de repérer les nouvelles lésions; une nouvelle ablation permet de soulager les symptômes.

\section{Introduction}

Interstitial cystitis (IC) is a clinical diagnosis characterized by urinary frequency and urgency, and pain in the absence of identifiable pathology. ${ }^{1,2}$ The International Continence Society (ICS) prefers the term "painful bladder syndrome" (PBS) to describe this symptom complex and reserves the diagnosis of IC for patients with "typical cystoscopic and histological features." ${ }^{11}$ Recent studies suggest that the prevalence of IC in the United States approaches 1 million people. ${ }^{3,4}$ The etiology of the disease is unclear and likely multifactorial. There have been published reports that implicate infection, immune dysregulation, mast cell infiltration, urothelial defects and/or neurologic mechanisms as causative factors. Treatment of patients with IC/PBS is as varied as the possible causative factors, and includes behavioural modification, oral medications, intravesical agents and surgical therapies. ${ }^{5,6}$

Hunner originally described a characteristic lesion or "ulcer" in some patients with interstitial cystitis which has led to the classification of IC into 2 types: ulcerative and non-ulcerative. ${ }^{7,8}$ The ulcerative type is estimated to occur in $\sim 10 \%$ of patients and may have a different pathophysiology than the non-ulcerative type. ${ }^{9}$ Hunner advocated surgical excision of ulcers to manage symptoms. More recently, endoscopic ablation has been reported to be successful. ${ }^{10}$ We report the outcomes of our patients with the ulcerative form of IC treated with endoscopic ablation.

\section{Methods}

Institutional review board approval was obtained. A retrospective chart review was performed on 14 patients, all female (mean age 63.6 years, range 28 to 85 ), treated in our clinic with cystoscopically identified bladder lesions, presumed to be Hunner's lesions, between 2003 and 2007. 
Payne et al.

Table 1. Number of other therapies attempted prior to flexible cystoscopy

\begin{tabular}{lc}
\hline No. of treatments & $\%$ of patients \\
\hline 1 & 7 \\
\hline 2 & 21 \\
\hline 3 & 21 \\
\hline 4 & 14 \\
\hline 5 & 37 \\
\hline $\begin{array}{l}\text { Five out of 14 patients had at least } 5 \text { other therapeutic interventions prior to endoscopic } \\
\text { ablation with 1 patient having had 10 unsuccessful therapies. }\end{array}$ \\
\hline
\end{tabular}

Patients had presented with the previous diagnosis of IC (made elsewhere) based on the characteristic symptoms (frequency, urgency and bladder pain) in the absence of other pathology (e.g., urinary tract infection, bladder cancer, stone). All patients had failed multiple standard therapies for IC prior to referral to our centre (Table 1) (Table 2). According to our routine PBS/IC protocol, flexible cystoscopy under local anesthesia (lidocaine jelly per urethra) was performed to identify the presence of Hunner's lesions. We considered any erythematous lesion as a potential Hunner's lesion if touching the lesion with the cystoscope reproduced the patient's symptoms (pain, urgency) and touching benign-appearing areas of urothelium did not. Often with these lesions, small vessels radiating toward a central area of pallor were noted, sometimes associated with a fibrinous exudate (Fig. 1). Owing to their symptoms and the
Table 2. Treatments attempted prior to referral

\begin{tabular}{lc}
\hline Treatment & No. of patients \\
\hline Anticholinergics & 14 \\
\hline Pentosan polysulfate & 11 \\
\hline Hydrodistention & 7 \\
\hline DMSO & 5 \\
\hline Antidepressants & 5 \\
\hline Prophylactic antibiotics & 3 \\
\hline Sacral neuromodulation & 2 \\
\hline Other* & 5 \\
\hline $\begin{array}{l}* \\
\text { benzor treatments including: silver nitrate instillation, hysterectomy, pyridium, }\end{array}$
\end{tabular}

identification of these lesions, all 14 patients met the National Institute of Diabetes and Digestive and Kidney Diseases (NIDDK) consensus research criteria for IC. ${ }^{11}$ At a later date, under a general anesthetic, the lesions were cystoscopically biopsied and ablated. Ablation was either by fulguration with a Bugbee electrode (12 patients) or formal resection with a loop cautery (2 patients). The decision to fulgurate versus resect was based on surgeon preference. Hydrodistension was avoided in all cases by limiting the amount of irrigation fluid instilled into the bladder to $200 \mathrm{~mL}$ and keeping the irrigation bag below $50 \mathrm{~cm}$ height. All cases were performed on an outpatient basis. Patients were seen at 1 month postoperatively, and then every 6 months, or if symptoms recurred.

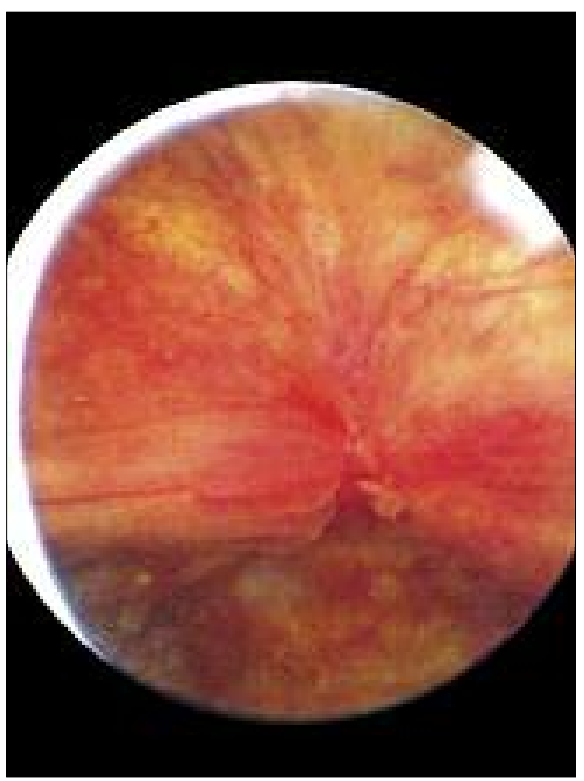

Fig. 1. Hunner's lesion identified on flexible cystoscopy.

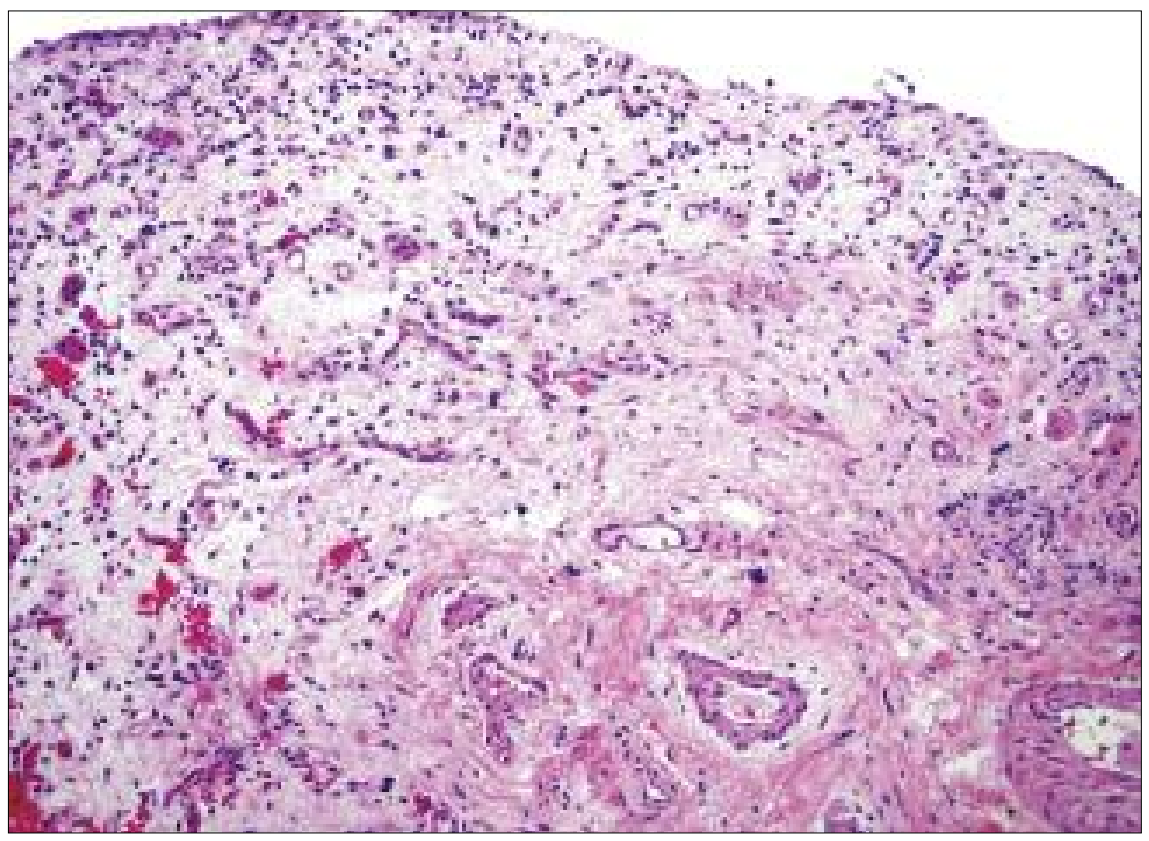

Fig. 2. Microscopy of biopsied Hunner's lesion demonstrating a mostly denuded surface with partial epithelization characterized by the presence of a monolayer of urothelium with umbrella cells (left upper corner). The underlying stroma is composed of edematous tissue with inflammatory cells (plasma cells, lymphocytes and rare mast cells). 


\begin{tabular}{|c|c|c|c|}
\hline & Prior to ablation $(n=14)$ & After ablation $(n=14)$ & $p$ \\
\hline Mean linear pain score $(0-10)$ & $8.1(95 \%$ Cl 6.5-9.8) & $1.7(95 \% \mathrm{Cl}-0.5-3.8)$ & 0.0002 \\
\hline No. of patients pain free & 0 & $7(50 \%)$ & $\mathrm{N} / \mathrm{A}$ \\
\hline$>50 \%$ improvement & N/A & $12(86 \%)$ & N/A \\
\hline No. of voids per $24 \mathrm{hr}$. & 23.5 (95\% Cl 16.5-30.6) & 13.5 (95\% Cl 5.8-21.2) & 0.014 \\
\hline
\end{tabular}

The primary outcome measures assessed were preoperative and postoperative (assessed 1 month post-operatively) analog pain score (patients were asked to grade the severity of their pain on a scale of 0 to 10 with 0 meaning no pain whatsoever and 10 meaning the worst pain imaginable), overall subjective improvement in symptoms (based on patients grading their percent level of improvement), change in urinary frequency on 48-hour bladder diary and bladder biopsy pathology reports.

\section{Results}

Following biopsy and ablation, 12 of 14 patients ( $86 \%$ ) experienced a substantial improvement in symptoms (Table 3 ). Two patients (14\%) experienced no change in their symptoms. The mean overall improvement in symptoms for these 14 patients was $76 \%$. All of the 12 patients who reported improvement had at least a $50 \%$ reduction in bladder pain and 7 reported complete resolution. The average linear pain scores decreased from 8.1 preoperatively to 1.7 postoperatively $(p=0.0002)$.

Nine patients (64\%) also reported subjective improvement in urinary frequency and urgency. Three of 12 respondents reported little or no improvement in frequency and urgency despite a dramatic decrease or complete resolution of bladder pain. The number of voids per day decreased from 23.5 preoperatively to 13.5 after treatment $(p=0.014)$. When assessing the 12 respondents, the number of voids per 24 hours decreased from 21.7 before treatment to 9.7 after treatment $(p=0.029)$.

Eight patients had a durable response and have not had a recurrence in their symptoms over an average follow-up of 27.8 months (range 8-55). Four patients experienced a return of their symptoms after a mean response of 12 months (range 7-23). In each of these 4 patients, symptomatic recurrence prompted repeat cystoscopy, which identified a recurrent lesion(s), typically in the region of a prior lesion. Re-treatment of the lesion(s) resulted in complete symptom resolution. One patient had a second recurrence 22 months after her first recurrence. Repeat treatment of the recurrent lesion with transurethral resection (TUR) resulted in complete symptom resolution. The average duration of response to a single treatment was 22.3 months. No complications resulted from endoscopic ablation.
The pathologic diagnosis was consistent with partial or complete epithelial denudation in 12 of the 14 specimens $(86 \%)$; chronic inflammation (predominantly lymphocytic and plasma cell infiltrates) was present in 13 of the 14 specimens (93\%), including all 12 patients who had symptomatic improvement. Figure 2 shows the microscopic appearance of a typical specimen. One of the 2 patients who did not benefit from endoscopic lesion ablation had a pathologic diagnosis of venous angioma with no evidence of inflammation. This patient was included in the analysis because, clinically, it was thought that this lesion was symptomatic (based on the office cystoscopy). However, pathologically, this was not a true case of a Hunner's lesion. Only 3 patients were noted to have mast cells in their pathologic specimen.

\section{Discussion}

In the early 1900s, Hunner carefully defined the characteristics of IC and identified bladder "ulcers" seen with this condition. ${ }^{7}$ More recently, Fall and colleagues recommended a change in nomenclature from "Hunner's ulcer" to "Hunner's lesion," because the bladder lesion is not a true ulcer. ${ }^{9}$ While Hunner initially recommended complete open excision of these lesions, ${ }^{7,8}$ most physicians abandoned this practice due to poor long-term results. ${ }^{12,13}$ However, with the advent of and improvements in endoscopic treatment, including TUR and laser ablation, the interest in treating these lesions returned. ${ }^{10,14-17}$ Peeker and colleagues reported improvement of symptoms in $90 \%$ of the 220 patients with IC treated with transurethral resection of Hunner's lesions. ${ }^{10}$ About half of those patients noted a durable (more than 3 years) response. Rofeim and colleagues had similarly good results using the Nd:YAG-laser to ablate Hunner's lesions. ${ }^{18}$ They noted that both pain and urgency appear to improve with laser ablation, but also noted that this treatment works best when the primary complaint is pain. Furthermore, they noted recurrent lesions in $46 \%$ of patients over an average follow-up of 23 months, but these patients responded to repeat treatment.

Our retrospective series confirms Peeker and Rofeim's data that local ablative therapy is effective in relieving symptoms in IC patients with Hunner's lesions. ${ }^{10,18}$ However, as was noted by Rofeim and colleagues, this is not neces- 
sarily a curative therapy, because lesions can recur. ${ }^{18}$ Nevertheless, patients experiencing recurrences appear to respond well to repeat treatment. Despite the growing evidence showing its potential effectiveness, endoscopic management remains an understudied and underutilized treatment option for this condition. In fact, in the most recent American Urological Association Update on PBS/IC from 2006, endoscopic resection or ablation of Hunner's lesions is not even listed as a potential treatment. ${ }^{6}$ However, our results, combined with others, show that endoscopic ablation of Hunner's lesions is effective and may be considered a first-line treatment for patients with ulcerative IC.

Peeker and others have proposed that removal or destruction of the intramural nerve endings engaged by the inflammatory process could be the mechanism of symptom relief. ${ }^{10,14}$ Whether this is done by fulguration or resection seems to be irrelevant, as both modalities were used in our patients with equivalent success. Recurrent lesions were noted in patients who had either fulguration or resection and typically occurred in the vicinity of a prior lesion. Whether complete, deep resection or even partial cystectomy, as originally advocated by Hunner, would prevent recurrence is unknown.

Biopsies of Hunner's lesions typically demonstrate a focal area of inflammation. Mast cells have been previously implicated as being an important and even necessary mediator of the inflammation associated with IC, with and without identification of Hunner's lesions on cystoscopy. ${ }^{10,13,19-21}$ Our pathologic analysis showed that most specimens had epithelial denudation with chronic inflammatory cells and a paucity of mast cells. Peeker and colleagues noted similar findings. ${ }^{10}$ Our biopsies tended to be superficial, often not including the deeper muscle where mast cell infiltration, if present, is typically noted. As a result, the absence of mast cells in our study may be due to sampling error. Regardless, it is still unclear whether the inflammatory aggregate seen on biopsy is the primary cause of symptoms or secondary to some other process that initiates the lesions.

We believe that office cystoscopy is important for diagnosing Hunner's lesions in patients with IC. We used lidocaine jelly per urethra and did not instill lidocaine intravesically, and found that our patients did not have difficulty tolerating flexible cystoscopy. Performing the procedure in an awake patient helps to determine if touching the bladder lesion with the cystoscope reproduces the patient's symptoms (while contact with normal appearing areas of the bladder does not). This confirmation seems to predict a favourable response to treatment with local ablative techniques. Since the diagnosis of IC is one of exclusion, any bladder lesion in a patient with symptoms of IC needs to be biopsied to exclude bladder cancer. There are reports of patients with a history of epithelial denudation on bladder biopsy (as was often noted in our cases) or IC subse- quently being diagnosed with transitional cell carcinoma or carcinoma in situ (CIS) of the bladder. ${ }^{22,23}$ We know of no evidence to suggest that Hunner's lesions may be associated with a risk of malignancy. The fact that these lesions can be mistaken for CIS gives us a low threshold to perform cystoscopy to identify urothelial pathology. Since IC is a chronic pain condition, we perform the biopsy and fulguration under an anaesthetic. We do not perform hydrodistension as this is not needed to diagnose the condition and generally does not provide significant symptomatic relief. ${ }^{24}$ During the time frame of our review, we performed 165 diagnostic cystoscopies on female patients with the symptoms of frequency/urgency and suprapubic/pelvic/bladder pain in whom no obvious pathologic cause for the symptoms was identified apart from the 14 patients in whom these lesions were identified. Excluding the 1 patient who was found pathologically to have a venous angioma, we had a prevalence of Hunner's lesion in about $8 \%$ of our $\mathrm{PBS} / \mathrm{IC}$ patients, which is consistent with the $10 \%$ prevalence that has been reported in the literature. ${ }^{9}$ While clearly not a common finding, the identification of these lesions is only possible via cystoscopic examination, and given the symptomatic improvement with endoscopic ablation that we and others have noted, we believe all patients with these symptoms warrant a cystoscopy.

One concern with repeated ablations is the potential for bladder fibrosis and ultimately an end-stage contracted bladder. Rofeim and colleagues recommended the use of the $\mathrm{Nd}$ :YAG-laser at a low power setting to minimize this risk. ${ }^{18}$ Although a legitimate concern, in our experience the identified Hunner's lesions have generally been focal and small ( $1 \mathrm{~cm}$ or less) patches, with 1 to 3 lesions per patient. This experience is not unlike what is seen in patients with recurrent superficial bladder cancer who potentially require repeated fulgurations and resections for cancer control. Bladder fibrosis in this setting has generally not been a problem. Bladder perforation is another concern, particularly when treating lesions at the dome, where the bladder wall tends to be thinnest. Use of a laser for ablation has not been immune to this, ${ }^{14}$ and thus no ablative modality is free from risk-caution is therefore needed when performing these procedures. By limiting distension of the bladder, one maximizes the bladder wall thickness, which may be protective. Whether laser ablation is any better or safer than cautery ablation would require a randomized study, which has yet to be performed.

Our study is clearly limited by its retrospective nature and the lack of a standardized IC symptom questionnaire evaluation, although a linear pain scale was used. Furthermore, deeper biopsy specimens that routinely included muscle might have helped to gain greater insight about the pathophysiology of these lesions. Follow-up endoscopy was only performed on patients with recurrent symptomatology and 
thus we do not know if lesions recurred in the absence of recurrent symptoms. Nevertheless, the improvement in symptoms in our patients following primary and repeat endoscopic ablation is noteworthy and consistent with the reports by Peeker and colleagues and Rofeim and colleagues. ${ }^{10,18}$

\section{Conclusion}

Hunner's lesions can be identified, through office cystoscopy, by their erythematous appearance and by the reproduction of the patient's symptoms upon touching the lesion with the cystoscope and failure of this effect upon touching normal-appearing urothelium. Endoscopic ablation of these lesions results in symptomatic improvement in most patients who have often failed prior therapies. Symptomatic recurrence can occur, and often responds to repeat ablation.

From the Medical College of Wisconsin, Milwaukee, WI

Competing interests: None declared.

This paper has been peer-reviewed.

\section{References}

1. Abrams $P$, Cardozo L, Fall M, et al. The standardisation of terminology of lower urinary tract function: report from the Standardisation Sub-committee of the International Continence Society. Neurourol Urodyn 2002;21:167-78.

2. Messing EM, Stamey TA. Interstitial cystitis: early diagnosis, pathology, and treatment. Urology 1978;12 381-92.

3. Curhan GC, Speizer FE, Hunter DJ, et al. Epidemiology of interstitial cystitis: a population based study. J Urol 1999;161:549-52.

4. Jones CA, Nyberg L. Epidemiology of intersititial cystitis. Urology 1997;49(5ASuppl):2-9.

5. Hanno P. Painful Bladder Syndrome/Interstitial Cystitis and Related Disorders. In: Wein AJ, et al., eds. Campbell-Walsh Urology. $9^{\text {th }}$ ed. Saunders Elsevier Philadelphia, 2007;330-70.
6. Zoltan E. FV, Hanno P. Painful bladder syndrome/Interstitial cystitis. AUA Update Series 2006;25: 78-87.

7. Hunner GL. A rare type of bladder ulcer in women; report of cases. Boston Med Surg J 1915;172:660-4.

8. Hunner GL. A rare type of bladder ulcer: further notes, with a report of eighteen cases. JAMA 1918;70: 203-12.

9. Fall M, Johansson SL, Aldenborg F. Chronic interstitial cystitis: a heterogeneous syndrome. J Urol 1987;137:35-8.

10. Peeker R, Aldenborg F, Fall M. Complete transurethral resection of ulcers in classic interstitial cystitis. Int Urogynecol J Pelvic Floor Dysfunct 2000;11:290-5.

11. Gillenwater JY, Wein AJ. Summary of the National Institute of Arthritis, Diabetes, Digestive and Kidney Diseases Workshop on Interstitial Cystitis, National Institutes of Health, Bethesda, Maryland, August 2829, 1987. J Urol 1988; 140:203-6.

12. Hand JR. Intersititial cystitis; report of 223 cases (204 women and 19 men). J Urol 1949;61:291-310.

13. Smith BH, Dehner LP. Chronic ulcerating interstitial cystitis (Hunner's ulcer). A study of 28 cases. Arch Pathol 1972;93:76-81.

14. Shanberg AM, Malloy T. Treatment of interstitial cystitis with neodymium: YAG laser. Urology 1987;29(4Suppl):31-3.

15. Fall M. Conservative management of chronic interstitial cystitis: transcutaneous electrical nerve stimulation and transurethral resection. J Urol 1985;133:774-8.

16. Greenberg E, Barnes R, Stewart S, et al. Transurethral resection of Hunner's ulcer. J Urol 1974;111:764-6.

17. Kerr WS, Jr. Interstitiial cystitis: treatment by transurethral resection. J Urol 1971;105:664-6.

18. Rofeim 0 , Hom D, Freid RM, et al. Use of the neodymium: YAG laser for interstitial cystitis: a prospective study. J Urol 2001;166:134-6.

19. Aldenborg $F$, Fall $M$, Enerback L. Proliferation and transepithelial migration of mucosal mast cells in interstitial cystitis. Immunology 1986;58:411-6.

20. Frenz AM, Christmas TJ, Pearce FL. Does the mast cell have an intrinsic role in the pathogenesis of interstitial cystitis? Agents Actions 1994;41 (Spec No:C14-15).

21. Sant GR, Kempurai D, Marchand JE, et al. The mast cell in intersitital cystitis: role in pathophysiology and pathogenesis. Urology 2007;69(4Suppl):34-40.

22. Levi AW, Potter SR, Schoenberg MP, et al. Clinical significance of denuded urothelium in bladder biopsy. J Urol 2001;166:457-60.

23. Tissot WD, Diokno AC, Peters KM. A referral center's experience with transitional cell carcinoma misdiagnosed as interstitial cystitis. J Urol 2004;172:478-80.

24. Ottem DP, Teichman JM. What is the value of cystoscopy with hydrodistension for interstitial cystitis? Urology 2005;66:494-9.

Correspondence: Dr. Michael L. Guralnick, Department of Urology, Medical College of Wisconsin, 9200 W Wisconsin Avenue, Milwaukee, WI 53226; fax: 414-805-0771; mguralni@mcw.edu 